\title{
Estimating the Dietary Intake of Breastfeeding Preterm Infants
}

\author{
Sarah Greenslade ${ }^{1}$, Jacqueline Miller ${ }^{1}$, Emma Tonkin ${ }^{1}$, Peter Marshall ${ }^{2}$ and \\ Carmel T. Collins ${ }^{3,4,5, *}$
}

1 Nutrition and Dietetics, Faculty of Medicine, Nursing and Health Sciences, School of Health Sciences, Flinders University Adelaide, Adelaide, 5002, South Australia, Australia; E-Mails: gree0385@uni.flinders.edu.au (S.G.); Jacqueline.miller@flinders.edu.au (J.M.); emma.tonkin@flinders.edu.au (E.T.)

2 Department of Neonatal Perinatal Medicine, Flinders Medical Centre, Flinders University, Adelaide, 5001, South Australia, Australia; E-Mail: peter.marshall@health.sa.gov.au

3 Women's and Children's Health Research Institute, Adelaide, 5006, South Australia, Australia;

4 Discipline of Paediatrics, School of Paediatrics and Reproductive Health, The University of Adelaide, Adelaide, 5000, South Australia, Australia

5 Healthy Mothers, Babies \& Children, South Australian Health and Medical Research Institute, Adelaide, 5000, South Australia, Australia

* Author to whom correspondence should be addressed; E-Mail: carmel.collins@health.sa.gov.au; Tel.: +61-881-617-443; Fax: +61-882-390-267.

Academic Editors: Jane Scott and Colin Binns

Received: 8 April 2015 / Accepted: 12 May 2015 / Published: 20 May 2015

\begin{abstract}
Aim: To determine how accurately the daily prescribed feed volume ( $\mathrm{mL} /$ day) estimates the actual intake of breastfeeding preterm infants and to characterise the volume taken during a breastfeed at differing gestational and postmenstrual ages. Methods: A cross sectional study was conducted on preterm infants born $<37$ weeks gestation from two Australian neonatal units. To determine the volume taken in a 24-h period infants were weighed before and after each breastfeed. This volume was added to the charted intake to determine the total intake and then compared to the prescribed feed volume. Bland Altman analyses were used to assess the level of agreement between the two methods. Results: Fifty six infants were studied on 206 breastfeeding occasions. There was a small bias ( $27 \mathrm{mLs} /$ day) but large $95 \%$ limits of agreement ( -76 to $130 \mathrm{~mL} /$ day). The volume taken during a single breastfeed ranged from 0 to $101 \mathrm{~mL}$ (median $23 \mathrm{~mL}$, IQR 9 to
\end{abstract}


$31 \mathrm{~mL}$ ) and was greater in more mature infants. Conclusions: Using the prescribed feed volume to estimate total intake has limited clinical utility for the individual infant, however the relatively small bias means that it may be useful within a population or for comparison between groups in which population means are compared. There was a large variation in volume taken during a breastfeed across all gestational and postmenstrual ages.

Keywords: breastfeeding; diet; infant preterm; nutrition assessment

\section{Introduction}

Breastfeeding and human milk are widely acknowledged as the gold standard for infant feeding and recommended by peak bodies such as the American Academy of Pediatrics [1] and the World Health Organization (WHO) [2]. Human milk is especially important for preterm infants (infants born $<37$ weeks gestational age) as these are the smallest, most vulnerable infants. The use of human milk is associated with a reduction in the incidence of several debilitating diseases such as necrotising enterocolitis [3]; retinopathy of prematurity (both incidence and severity) [4,5]; late onset sepsis [6]; and infection [7]. Human milk also confers significant neuro developmental benefit to preterm infants [8,9].

In addition to the quality of milk provided, it is critical for preterm infants to receive an adequate volume of milk to optimise growth and developmental outcomes. Even small variations in volume can have a significant impact on the dietary adequacy for very small infants and hence there is a need for accurate methods of measuring dietary intake [10]. Guidelines for nutritional management have been published, [11] however benchmarking against them is difficult when infants are transitioning from tube to breast feeds. During this transition, intake data accounting for both the contribution from tube and breast feeds are limited [12-15]. This is in contrast to early in the admission when feeds are gavaged or when fed by bottle and intake can be accurately recorded, and calorie and protein intakes can be calculated and documented. In-hospital breastfeeding experience is essential to aid the transition to full breastfeeding by discharge; however measuring the volume taken at a breastfeed is problematic.

The most commonly used method for estimating breastfeed volume in research reporting dietary intake of preterm infants is calculation of the prescribed feed volume minus the charted intake, with the remainder assumed to be the breastfeed volume [16]. Other approaches taken include ignoring the contribution from breastfeeds [12-15], using complex statistical approaches to impute the volume [17] or using the test weighing method [18-20]. Test weighing involves weighing infants before and after a breastfeed, with the change in weight reflecting the milk volume taken by the infant. Test weighing is considered the gold standard for measuring volume taken during a breastfeed in a clinical setting [10,19,21,22]. However, it is not routinely used in practice due to the perceived impact it may have on mothers' confidence with breastfeeding [20,23] and the burden it places on staff. Rather, nutritional adequacy is most commonly assessed by monitoring weight gain.

The aim of this study was to determine if the commonly used proxy, prescribed feed volume, can accurately estimate the actual intake of breastfeeding preterm infants and therefore be reliably used when reporting dietary intake data in nutritional audits. A secondary aim was to characterise the volume of milk taken during a breastfeed according to gestational (GA) and postmenstrual age (PMA). 


\section{Methods}

\subsection{Design}

This cross sectional study compared the prescribed feed volume of preterm infants with their actual intake. Actual intake was calculated using the test weighing method at each breastfeed during 24-h periods, combined with the measured enteral intake from fluid balance charts. The primary care physician prescribed the feed volume which was documented daily on the infant's care plan and delivered through breast, bottle or gavage tube feeding. GA is defined as the period of time between the first day of the last menstrual period to the day of delivery [24]. PMA is GA plus the time elapsed after birth (chronological age) [24].

\subsection{Participants}

Mother-infant dyads were recruited from the neonatal units of Flinders Medical Centre (FMC) and Lyell McEwin Hospital (LMH) Adelaide, South Australia from June to November 2013. FMC is one of the two neonatal intensive care units in South Australia with 11 intensive care and 24 special care cots, while LMH is a 20 cot Special Care facility. Both units support non-critically ill infants transitioning to suck feedings and therefore provide an ideal population group for the study. Participating infants met the following inclusion criteria: born $<37$ weeks gestation; at least 5 days old to allow adequate time for mothers' milk supply to come in [25]; PMA between 32-40 weeks as coordinated suck swallow is typically observed no earlier [26]; and taking some breastfeeds. The study excluded infants whose medical instability or clinical condition prevented test weighing or impacted oral intake.

Infant characteristics such as sex, GA, PMA, birth anthropometrics, birth plurality, and ethnicity were collected from medical records. Nutritional information including the prescribed feed volume (daily total $\mathrm{mL} / \mathrm{kg} /$ day and $\mathrm{mL} /$ feed) and feed frequency were collected from the infants' care plans. Infants were weighed daily or second daily under standard conditions and this weight was used by the clinicians to calculate the daily prescribed feed volume ( $\mathrm{mL} /$ day). The fluid balance charts were reviewed for each 24-h period to determine the total volume taken via nasogastric tube or bottle.

All parents/guardians gave their informed consent for inclusion before their infant participated in the study. The study was conducted in accordance with the Declaration of Helsinki, and the protocol was approved by the Southern Adelaide Clinical Human Research Ethics Committee (ID 224.1).

\subsection{Test Weighing}

The test weighing procedure was adapted from the method described by Hasse, et al. [21] for preterm and high risk hospitalised infants. Briefly, the clothed infant was weighed directly before and immediately after a breastfeed under the same conditions. Both the pre and post feed weights were obtained twice, and the average of the two measurements used, in order to minimise measurement error. If the discrepancy between the two weights was more than $5 \mathrm{~g}$, the weighing procedure was repeated until the discrepancy was $\leq 5 \mathrm{~g}$. Infants were weighed using SECA-727 (SEcac, Hamburg, Germany) scales at FMC and Tanita BLB-12 (Tanita, Tokyo, Japan) scales at LMH, both of which are sensitive to $2 \mathrm{~g}$ [27]. A researcher was present for all measurements to confirm adherence to the study protocol. Experienced neonatal nursing 
staff placed the infants on the scale, while the researcher read the digitally recorded weight. This ensured clinical staff and parents remained blind to the test weight results, preventing interference with feeding management and avoiding any potential stress with breastfeeding and test weighing. Infants staying in the unit for more than a few days could have the test weighing procedure repeated twice weekly. Results from the test weigh were deemed invalid if there was a negative weight change that was beyond the standard error of the scales ( $\pm 2 \mathrm{~g}$ ). Furthermore if a breastfeed within the 24 -h period was not measured, that $24-\mathrm{h}$ period record was deemed invalid for the primary aim.

\subsection{Statistical Analysis}

A Bland-Altman assessment for agreement was used to compare the actual intake and the prescribed feed volume ( $\mathrm{mL} /$ day) for estimating breastfeed volume [28,29]. The range of agreement was defined as mean bias \pm 2 standard deviations (SD). To estimate the Limits of Agreement (LoA) with acceptable accuracy, it was determined a sample of 100 measurements (100 24-h dietary intake records) were required [29]. Bland-Altman plots were repeated for infants receiving $\leq 2$ breastfeeds and $>2$ breastfeeds over the $24-\mathrm{h}$ period to investigate if the number of breastfeeds influenced the level of agreement between the two methods. The LoA method assumes independent observations i.e., that each pair of measurements is from a different individual. However, the originators of the LoA method, Bland and Altman, acknowledge that multiple measurements per individual are often undertaken in clinical settings and have described methods for analysing repeat data [30]. If the within subject variance is larger than or close to the between subject variance then the LoA are more likely to be close to the values obtained if the data were all truly independent. Conversely, if the within subject variance is less than the between subject variance then the LoA will be narrower, suggesting that there was overestimation of agreement between the two methods. Thus, the within and between subject variation was compared by determining the mean bias, standard deviation and the $95 \%$ limits of agreement. The secondary aims explored if the volume of milk taken at a breastfeed varied according to GA and PMA. Infants born at earlier GA are typically smaller with a more complex medical progression; therefore the intake data were descriptively examined for differences in GA by separating infants into 2-week GA categories. Additionally, as it was expected that more mature infants would have a better coordinated suck-swallow-breathe pattern [31,32], intake data (averaged for each 24-h period

per infant) were descriptively examined by PMA (in weeks). IBM SPSS version 19.0 (IBM Corp., Armonk, NY, USA) statistical software was used for statistical analyses.

\section{Results}

Fifty-nine preterm infants were enrolled in the study. The mean birth weight was $2123 \mathrm{~g}$ (SD 499), with median GA of 34 weeks (minimum 27 weeks, maximum 36 weeks) (Table 1). Infants were test weighed on average twice, with a minimum of 1 and maximum of 9 times per infant. The number of breastfeeds, and therefore test weighing procedures, within a 24-h period ranged between 1 and 4 with a median of 2 . The mean prescribed feed volume was $386 \mathrm{~mL}, \mathrm{SD} 73$. 
Table 1. Infant baseline characteristics.

\begin{tabular}{ll}
\hline Baseline Characteristics & $\begin{array}{l}\text { Infants } \\
\mathbf{N}=\mathbf{5 9} \\
\mathbf{N}(\mathbf{\%})\end{array}$ \\
\hline Recruiting centre & \\
Flinders Medical Centre & $25(42)$ \\
The Lyell McEwin Hospital & $34(58)$ \\
Male sex & $32(54)$ \\
Plurality & \\
Singleton & $39(66)$ \\
Twins & $20(34)$ \\
Birth weight, $g$ (mean, SD) & $(2123,499)$ \\
Gestational age, weeks (median, min, max) & $(34,27,36)$ \\
\hline
\end{tabular}

\subsection{Primary Outcome}

Three infants were excluded from the primary method validation analysis because of invalid test weighs within the 24-h measuring period. Of the remaining 56 infants, 26 were measured repeatedly, resulting in 103, 24-h dietary intake records. The Bland-Altman LoA plot comparing the actual intake with the prescribed feed volume is presented in Figure 1. The mean difference (or bias) between the infants' prescribed feed volume and actual intake was $27 \mathrm{~mL} / \mathrm{day}, \mathrm{SD} 53 ; 95 \% \mathrm{LoA}-76$ to $130 \mathrm{~mL} /$ day. Thus the prescribed feed volume systematically overestimated actual intake by a mean of $27 \mathrm{~mL} /$ day, representing 7\% of the mean prescribed feed volume. Using the method described by Bland and Altman to account for repeated measures [30] the mean bias remains the same $(27 \mathrm{~mL} /$ day, SD 54; $95 \%$ LoA -79 to $133 \mathrm{~mL} /$ day). Given that the within subject variance was marginally larger than the between variance, for the purpose of this analysis each measurement can be treated as independent. The bias was less when infants received $\leq 2$ breastfeeds daily $(16 \mathrm{~mL} /$ day, 95\% LoA -76 to $107 \mathrm{~mL} /$ day), indicating closer agreement between the two methods (Figure 2). The bias was larger with $>2$ breastfeeds a day ( $72 \mathrm{~mL} /$ day, $95 \%$ LoA -32 to $175 \mathrm{~mL} /$ day; Figure 3$)$.

\subsection{Secondary Outcomes}

All 59 infants were included in the secondary analyses investigating breast milk intake at differing GA and PMA. A total of 240 individual breastfeeding sessions were measured with 32 infants measured on more than one occasion. Five measurements were excluded because of negative results beyond the standard error of the scales, leaving a total of 235 measurements for the analyses. The median intake for a single breastfeeding session across all GA categories was $23 \mathrm{~mL}$ (IQR 9 to $31 \mathrm{~mL}$ ) and the volume taken at a single breastfeed ranged from 0 to $101 \mathrm{~mL}$ (Table 2). Six percent $(\mathrm{N}=14)$ of total measured breastfeeds recorded no nutritional intake (i.e., no weight gain was observed) with a further $25 \%(\mathrm{~N}=60)$ with an intake of $10 \mathrm{~mL}$ or less (Figure 4). The volume taken from breastfeeding accounted for $12 \%$ of the daily intake. The median volume taken during a single breastfeed increased with increasing PMA (Table 3). 


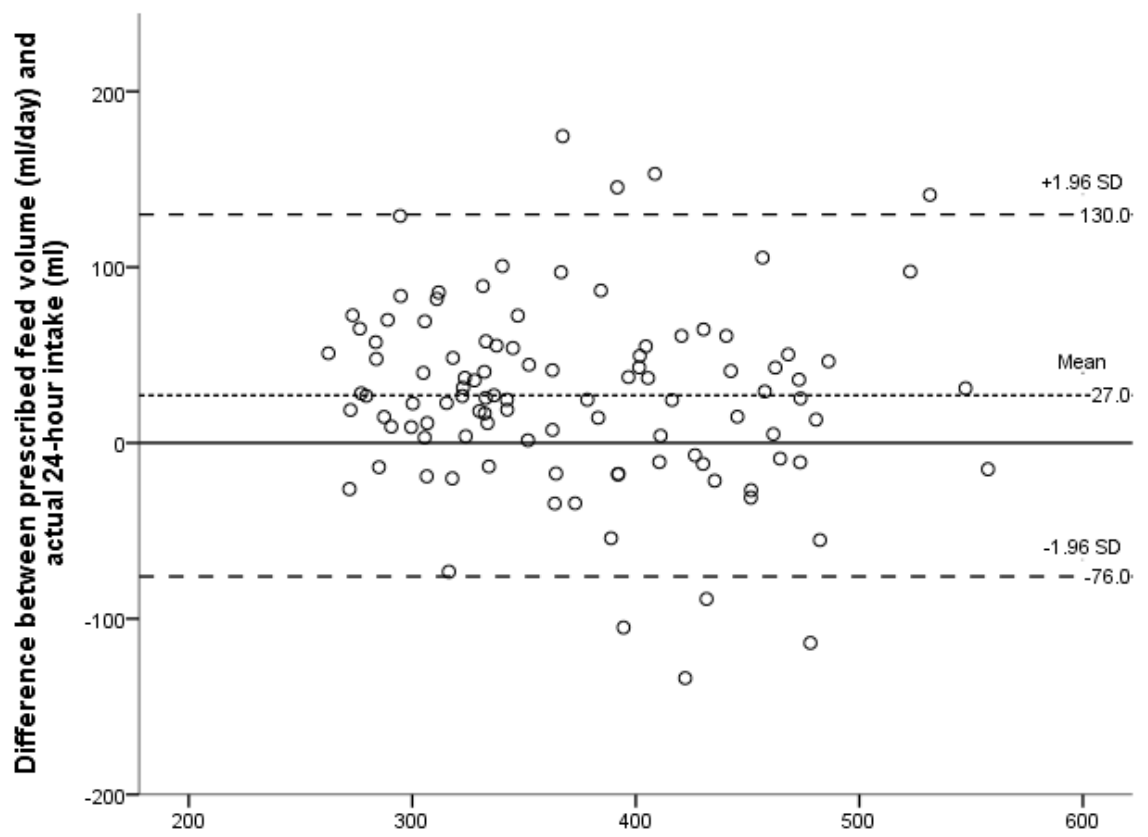

Average of prescribed feed volume ( $\mathrm{ml} / \mathrm{day}$ ) and actual 24-hour intake ( $\mathrm{ml})$

Figure 1. Bland-Altman plot of the agreement between the prescribed feed volume in $\mathrm{mL} /$ day and the actual 24-h intake of the infant; each point represents one infant's intake over $24 \mathrm{~h}(\mathrm{~N}=103)$.

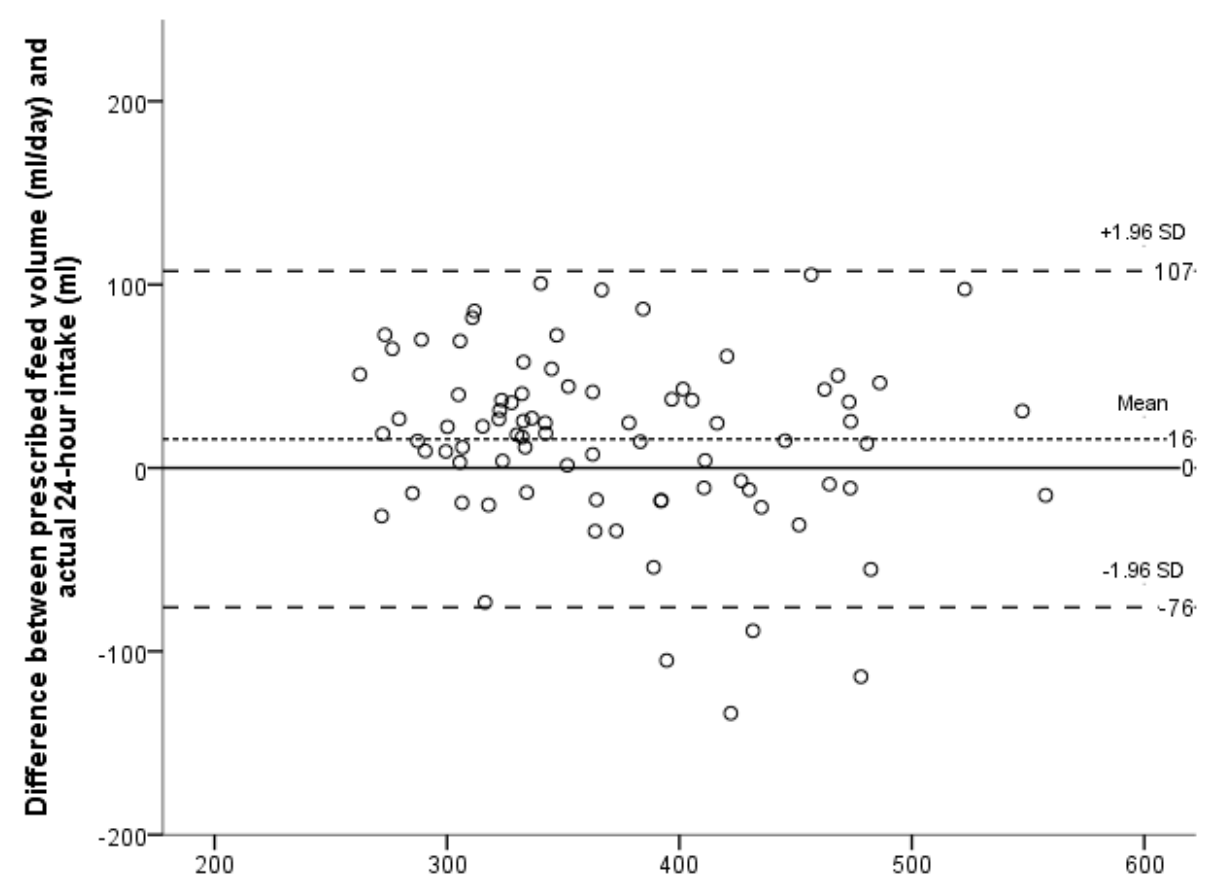

Average of prescribed feed volume (ml/day) and actual 24-hour intake (ml)

Figure 2. Bland-Altman plot of the agreement between the prescribed feed volume in $\mathrm{mL} / \mathrm{day}$ and the actual intake of the infant for infants receiving $\leq 2$ breastfeeds in $24 \mathrm{~h}$. Each point represents one infant's intake over $24 \mathrm{~h}$ for infants receiving $\leq 2$ breastfeeds $(\mathrm{N}=83)$. 


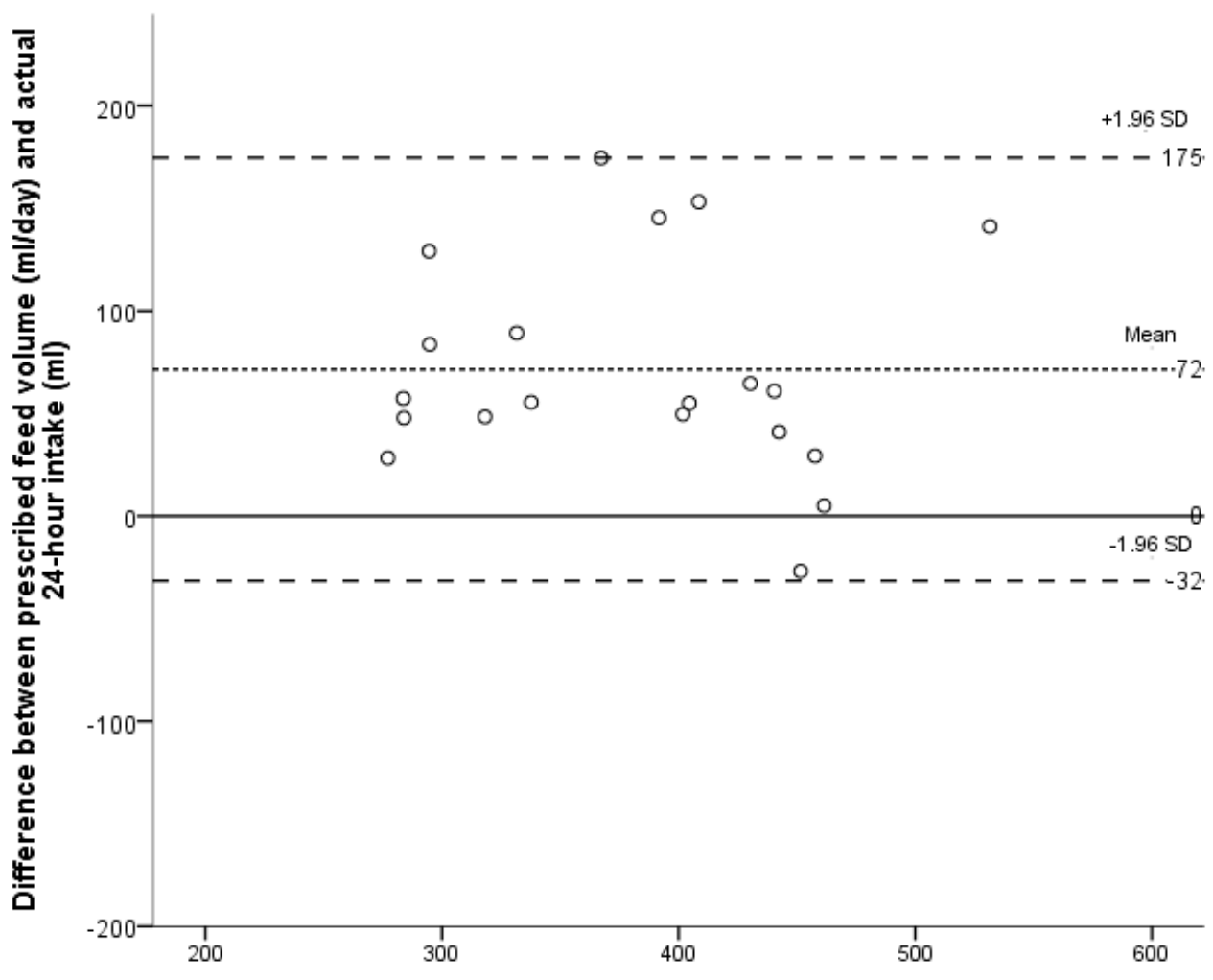

Average of prescribed feed volume ( $\mathrm{ml} / \mathrm{day})$ and actual 24-hour intake ( $\mathrm{ml}$ )

Figure 3. Bland-Altman plot of the agreement between the prescribed feed volume in $\mathrm{mL} /$ day and the actual intake of the infant for infants receiving $>2$ breastfeeds in $24 \mathrm{~h}$. Each point represents one infant's intake over $24 \mathrm{~h}$ for infants receiving $>2$ breastfeeds $(\mathrm{N}=20)$.

Table 2. Median breast milk intake (mLs) per breastfeed according to gestational age (GA) at birth.

\begin{tabular}{ccccc}
\hline GA (Weeks) & Infants, $N$ & Breast Feeds, N & Volume * $(\mathbf{m L})$ & Min, Max $(\mathbf{m L})$ \\
\hline $27-28$ & 3 & 19 & $23(4,31)$ & 0,86 \\
$29-30$ & 4 & 20 & $12(9,30)$ & 1,52 \\
$31-32$ & 8 & 32 & $13(5,26)$ & 0,48 \\
$33-34$ & 19 & 83 & $26(7,34)$ & 0,101 \\
$35-36$ & 25 & 81 & $24(17,34)$ & 0,87 \\
\hline \multicolumn{5}{c}{ * volume $(\mathrm{mL})$ reported as median and IQR }
\end{tabular}

* volume $(\mathrm{mL})$ reported as median and IQR. 


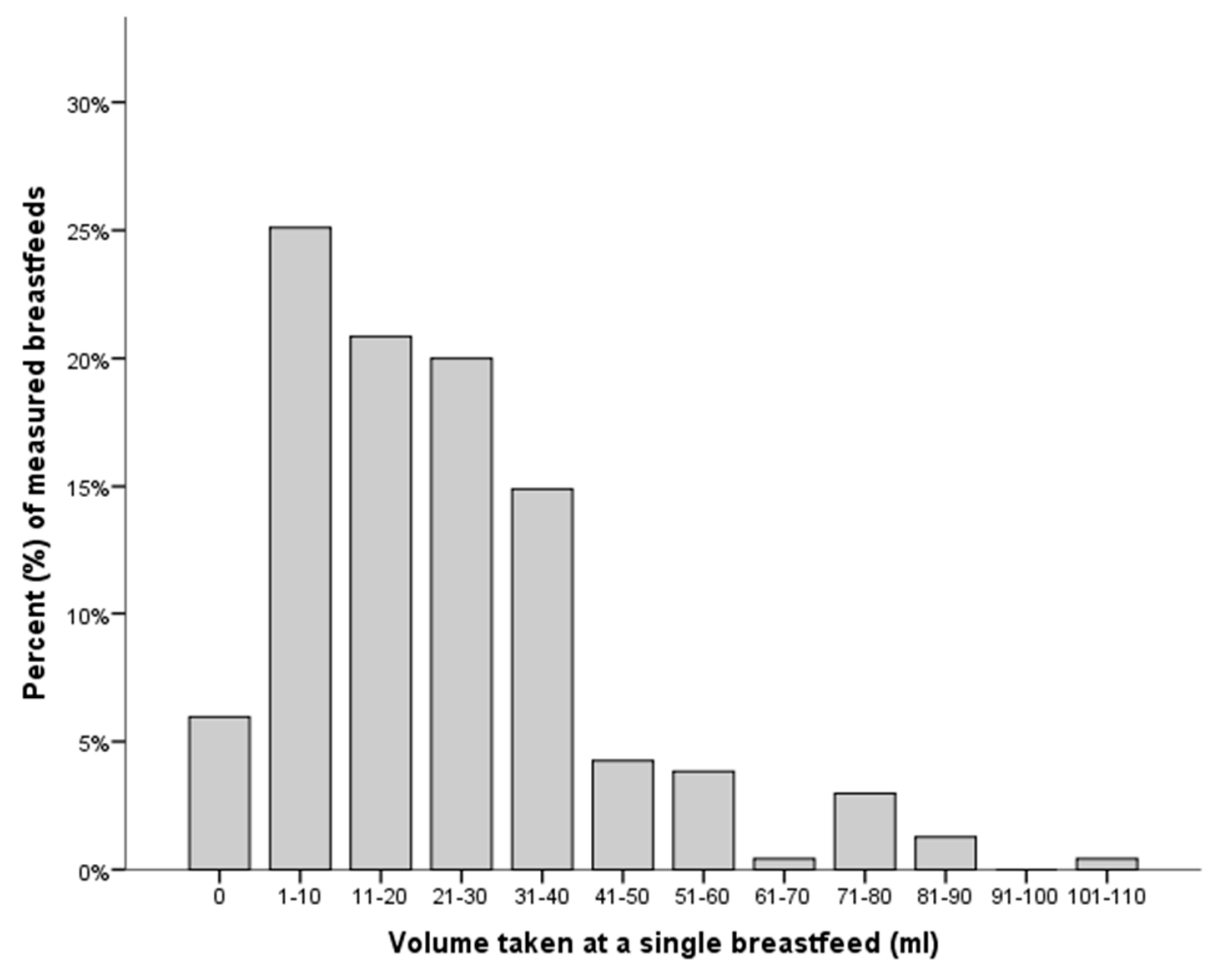

Figure 4 The percentage (\%) of breast milk (mLs) taken during a single breastfeed in $10 \mathrm{~mL}$ increments.

Table 3. Median breast milk intake (mLs) per breastfeed according to post menstrual age (PMA).

\begin{tabular}{cccc}
\hline PMA (Weeks) & N Infants & $\begin{array}{c}\text { Volume } \\
(\mathbf{m L})\end{array}$ & $\begin{array}{c}\text { Min, Max } \\
(\mathbf{m L})\end{array}$ \\
\hline 32 & 2 & $15 *$ & 1,30 \\
33 & 6 & $9(4-17)$ & 2,32 \\
34 & 10 & $18(8-32)$ & 2,56 \\
35 & 26 & $12(5-26)$ & 0,64 \\
36 & 46 & $26(14-34)$ & 0,87 \\
37 & 20 & $21(6-30)$ & 0,76 \\
$38+$ & 11 & $36(28-43)$ & 0,101 \\
\hline
\end{tabular}

Volume $(\mathrm{mL})$ reported as median and IQR; * IQR range not calculated due to small number

\section{Discussion}

The prescribed feed volume overestimates the dietary intake of preterm infants transitioning to full sucking breastfeeds. While the overestimation is relatively modest, due to the wide limits of agreement the prescribed feed volume is poor at estimating actual intake at the individual infant level. The volume taken during a breastfeed was highly variable however, as would be expected, there was an overall trend to an increased volume of intake with increasing PMA. We elected not to test for statistical differences in volume taken according to either GA or PMA as some infants were measured 
only once, while others had multiple observations, thereby invalidating the statistical assumptions of independence for both the Kruskal-Wallis and Friedman tests.

On an individual level the prescribed feed volume will over or under estimate intake by $+/-103 \mathrm{~mL}$ (Figure 1). To place this in context, the infant whose actual intake was overestimated by $174 \mathrm{~mL}$ (Figure 1) was prescribed $180 \mathrm{mLs} / \mathrm{kg} /$ day (daily total of $455 \mathrm{~mL}$ ) and therefore theoretically missed out on $40 \%$ of the daily intake, or in this particular case 2 of the 6 prescribed daily feeds.

We found that as the number of breastfeeds increased the extent to which the prescribed feed volume overestimated the actual intake increased (Figure 3). This suggests that in practice the volume that preterm infants are taking at a breastfeed is overestimated and, therefore, with increasing number of breastfeeds, the gap between estimated and actual intake is compounded. This is further confirmed by the fact that the median intake at a single breastfeed was $23 \mathrm{~mL}$, notably less than the median prescribed per feed of $60 \mathrm{~mL}$.

In this study a large variation in volume taken during a breastfeed across all GAs and PMAs was observed (Table 2 and Table 3). It is worthwhile highlighting that $6 \%$ of all measured breastfeeds had zero intake and $25 \%$ of feeds only measured $1-10 \mathrm{~mL}$. This zero to minimal intake was observed across all GAs and PMAs. This is similar to the findings by Meier et al. [18-20] who reported occasions where there was minimal to no nutritive intake during a breastfeed. As expected more mature infants had a greater intake, with the average intake at a single feed greater in the older infants. This result likely reflects that infants are typically larger and more mature at later PMA.

For dietary audits it would be reasonable to use the prescribed feed volume to describe the dietary intake of breastfeeding preterm infants at a population level as the overestimate represents $7 \%$ of the mean prescribed feed volume for our population of infants. Furthermore considering that on average $12 \%$ of the actual intake $(\mathrm{mL})$ was from breastfeeds, using the prescribed feed volume would improve the accuracy of audits that do not account for the contribution from breastfeeds [12-15].

A strength of this study was that the sample size was adequate to ensure the measures of agreement were calculated with reasonable precision. The test weighing procedure was adapted from a technique developed for preterm and high-risk hospitalised infants [21], thus protocols used were designed to avoid common reasons for measurement error specifically in this population. The study was conducted across two neonatal units, allowing for more generalizable results across the preterm population.

Participating infants' GA ranged from 27 to 36 weeks, however the earlier GA were under represented and future studies would benefit from collecting dietary intake data from infants in the earlier GA categories (27-32 weeks). While the sample size was adequate for the primary aim of the study, it was insufficient to make inferences about the average intake of preterm infants.

\section{Conclusions}

In dietary audits, the volume of intake of breastfeeding preterm infants can be estimated with reasonable accuracy at a population level using the prescribed feed volume. However use of prescribed feed volume cannot be recommended as a useful clinical tool for individual infants. There is a large variation in the volume of milk taken during a breastfeed across all gestational and postmenstrual ages, with the overall intake higher in more mature infants. 


\section{Acknowledgments}

We would like to thank the families who so generously participated in this study, the nursing and medical staff at the Flinders Medical Centre and Lyell McEwin Hospital neonatal units and Ms. Rachel Battersby for her help with data collecting. This work was supported by the Women's and Children's Health Research Institute, the University of Adelaide; Nutrition and Dietetics, Flinders University and the Australian National Health and Medical Research Council (NHMRC) Centre of Research Excellence "Foods for Future Australians" (APP1035530). CTC is supported through a McLeod Postdoctoral Research Fellowship (MS McLeod Research Fund, Women's and Children's Hospital Research Foundation).

\section{Author Contributions}

Carmel Collins, Jacqueline Miller. and Sarah Greenslade conceived and designed the study; Sarah Greenslade performed the study; Sarah Greenslade, Emma Tonkin, Jacqueline Miller. and Carmel Collins analysed the data; Peter Marshall contributed materials and facilitated access to patients; Sarah Greenslade drafted the manuscript; all authors contributed to the final manuscript.

\section{Conflicts of Interest}

The authors declare no conflict of interest. The funding bodies had no role in the design of the study; in the collection, analyses, or interpretation of data; in the writing of the manuscript, and in the decision to publish the results. The contents of the published material are solely the responsibility of the authors and do not reflect the views of the NHMRC.

\section{References}

1. American Academy of Pediatrics. Breastfeeding and the use of human milk. Pediatrics 2012, 129, 827-841.

2. Global Nutrition Targets 2025: Breastfeeding Policy Brief (WHO/NHM/NHD/14.7); World Health Organization: Geneva, Switzerland, 2014.

3. Schanler, R.J.; Shulman, R.J.; Lau, C.; Smith, E.O.B.; Heitkemper, M.M. Feeding strategies for premature infants: Randomized trial of gastrointestinal priming and tube-feeding method. Pediatrics 1999, 103, 434-439.

4. Manzoni, P.; Stolfi, I.; Pedicino, R.; Vagnarelli, F.; Mosca, F.; Pugni, L.; Bollani, L.; Pozzi, M.; Gomez, K.; Tzialla, C.; et al. Human milk feeding prevents retinopathy of prematurity (rop) in preterm vlbw neonates. Early Hum. Dev. 2013, 89, 64-68.

5. Hylander, M.A.; Strobino, D.M.; Pezzullo, J.C.; Dhanireddy, R. Association of human milk feedings with a reduction in retinopathy of prematurity among very low birthweight infants. Amer. J. Perinatol. 2001, 21, 356-362.

6. Furman, L.; Taylor, G.; Minich, N.; Hack, M. The effect of maternal milk on neonatal morbidity of very low-birth-weight infants. Arch. Pediatr. Adolesc. Med. 2003, 157, 66-71.

7. Hylander, M.A.; Strobino, D.M.; Dhanireddy, R. Human milk feedings and infection among very low birth weight infants. Pediatrics 1998, doi:10.1542/peds.102.3.e38. 
8. Vohr, B.R.; Poindexter, B.B.; Dusick, A.M.; McKinley, L.T.; Higgins, R.D.; Langer, J.C.; Poole, W.K. Persistent beneficial effects of breast milk ingested in the neonatal intensive care unit on outcomes of extremely low birth weight infants at 30 months of age. Pediatrics 2007, 120, 953-959.

9. Vohr, B.R.; Poindexter, B.B.; Dusick, A.M.; McKinley, L.T.; Wright, L.L.; Langer, J.C.; Poole, W.K. Beneficial effects of breast milk in the neonatal intensive care unit on the developmental outcome of extremely low birth weight infants at 18 months of age. Pediatrics 2006, 118, 115-123.

10. Scanlon, K.S.; Alexander, M.P.; Serdula, M.K.; Davis, M.K.; Bowman, B.A. Assessment of infant feeding: The validity of measuring milk intake. Nutr. Rev. 2002, 60, 235-251.

11. McGuire, W.; Henderson, G.; Fowlie, P.W. Feeding the preterm infant. BMJ 2004, 329, 1227-1230.

12. Cooke, R.J.; Embleton, N.E.; Pang, N. Postnatal malnutrition and growth retardation: An inevitable consequence of current recommendations in preterm infants? Pediatrics 2001, 2001, 270-273.

13. Cormack, B.E.; Bloomfield, F.H. Audit of feeding practices in babies $<1200 \mathrm{~g}$ or 30 weeks gestation during the first month of life. J. Paediatr. Child Health 2006, 42, 458-463.

14. Ernst, K.D.; Radmacher, P.G.; Rafail, S.T.; Adamkin, D.H. Postnatal malnutrition of extremely low birth-weight infants with catch-up growth postdischarge. J. Perinatol. 2003, 23, 477-482.

15. Radmacher, P.G.; Looney, S.W.; Rafail, S.T.; Adamkin, D.H. Prediction of extrauterine growth retardation (eugr) in vvlbw infants. J. Perinatol. 2003, 23, 392-395.

16. McLeod, G.; Sherriff, J.; Nathan, E.; Hartmann, P.E.; Simmer, K. Four-week nutritional audit of preterm infants born $<33$ weeks gestation. J. Paediatr. Child Health 2013, 49, 332-339.

17. Collins, C.T.; Chua, M.C.; Rajadurai, V.S.; McPhee, A.J.; Miller, L.N.; Gibson, R.A.; Makrides, M. Higher protein and energy intake is associated with increased weight gain in pre-term infants. $J$. Paediatr. Child Health 2010, 46, 96-102.

18. Meier, P.P.; Brown, L.P.; Hurst, N.M.; Spatz, D.L.; Engstrom, J.L.; Borucki, L.C.; Krouse, A.M. Nipple shields for preterm infants: Effect on milk transfer and duration of breastfeeding. J. Hum. Lact. 2000, 16, 106-114.

19. Meier, P.P.; Engstrom, J.L.; Crichton, C.L.; Clark, D.R.; Williams, M.M.; Mangurten, H.H. A new scale for in-home test-weighing for mothers of preterm and high risk infants. J. Hum. Lact. 1994, 10, 163-168.

20. Meier, P.P.; Engstrom, J.L.; Fleming, B.A.; Streeter, P.L.; Lawrence, P.B. Estimating milk intake of hospitalized preterm infants who breastfeed. J. Hum. Lact. 1996, 12, 21-26.

21. Hasse, B.; Barreira, J.; Murphy, P.K.; Mueller, M.; Rhodes, J. The development of an accurate test weighing technique for preterm and high-risk hospitalized infants. ABM 2004, 4, 151-156.

22. Meier, P.P.; Lysakowski, T.Y.; Engstrom, J.L.; Kavanaugh, K.L.; Mangurten, H.H. The accuracy of test weighing for preterm infants. J. Pediat. Gastroenterol. Nutr. 1990, 10, 62-65.

23. Hall, W.A.; Shearer, K.; Mogan, J.; Berkowitz, J. Weighing preterm infants before \& after breastfeeding: Does it increase maternal confidence and competence? MCN 2002, 27, 318-326.

24. Committee on Fetus and Newborn. Age terminology during the perinatal period. Pediatrics 2004, 114, 1362-1364.

25. Scott, J.A.; Binns, C.W.; Oddy, W.H. Predictors of delayed onset of lactation. Matern. Child Health J. 2007, 3, 186-193. 
26. Fucile, S.; Gisel, E.; Lau, C. Oral stimulation accelerates the transition from tube to oral feeding in preterm infants. J. Pediatrics 2002, 141, 230-236.

27. Riordan, J.; Wambach, K. Breastfeeding and Human Lactation, 4th ed.; Jones and Bartlett: Sudbury, MA, USA, 2010.

28. Bland, J.M.; Altman, D.G. Statistical methods for assessing agreement between two methods of clinical measurement. Lancet 1986, 1, 307-310.

29. Frequently Asked Questions on the Design and Analysis of Measurement Studies. Available online: http://www-users.york.ac.uk/ mb55/meas/comfaq.htm (accessed on 18 December 2014).

30. Bland, J.M.; Altman, D.G. Agreement between methods of measurement with multiple observations per individual. J. Biopharm. Stat. 2007, 17, 571-582.

31. Dodrill, P.; Donovan, T.; Cleghorn, G.; McMahon, S.; Davies, P.S. Attainment of early feeding milestones in preterm neonates. Amer. J. Perinatol. 2008, 28, 549-555.

32. Fucile, S.; Gisel, E.; Schanler, R.J.; Lau, C.A. A controlled-flow vacuum-free bottle system enhances preterm infants' nutritive sucking skills. Dysphagia 2009, 24, 145-151.

(C) 2015 by the authors; licensee MDPI, Basel, Switzerland. This article is an open access article distributed under the terms and conditions of the Creative Commons Attribution license (http://creativecommons.org/licenses/by/4.0/). 